\title{
Article \\ The Efficacy and Predictability of Maxillary First Molar Derotation with Invisalign: A Prospective Clinical Study in Growing Subjects
}

\author{
Roberta Lione ${ }^{1,2, *(\mathbb{D})}$, Valeria Paoloni ${ }^{1}$, Francesca Chiara De Razza ${ }^{1}$, Chiara Pavoni ${ }^{1}$ (D) and Paola Cozza ${ }^{1}$ \\ 1 Department of Systems Medicine, University of Rome Tor Vergata, 00133 Rome, Italy; \\ vamapa87@gmail.com (V.P.); francescachiara.derazza@outlook.it (F.C.D.R.); \\ dott.chiarapavoni@gmail.com (C.P.); profpaolacozza@gmail.com (P.C.) \\ 2 Department of Systems Medicine, Saint Camillus International University of Health Sciences, \\ 00131 Rome, Italy \\ * Correspondence: robertalione@yahoo.it
}

check for updates

Citation: Lione, R.; Paoloni, V.; De Razza, F.C.; Pavoni, C.; Cozza, P. The Efficacy and Predictability of Maxillary First Molar Derotation with Invisalign: A Prospective Clinical Study in Growing Subjects. Appl. Sci. 2022, 12, 2670. https:// doi.org/10.3390/app12052670

Academic Editors: Simone Parrini and Dorina Lauritano

Received: 30 January 2022

Accepted: 1 March 2022

Published: 4 March 2022

Publisher's Note: MDPI stays neutral with regard to jurisdictional claims in published maps and institutional affiliations.

Copyright: (c) 2022 by the authors. Licensee MDPI, Basel, Switzerland. This article is an open access article distributed under the terms and conditions of the Creative Commons Attribution (CC BY) license (https:// creativecommons.org/licenses/by/ $4.0 /)$.

\begin{abstract}
To analyze the derotation of maxillary mesio-rotated first permanent molars in subjects with Class II edge-to-edge dental malocclusion in mixed dentition treated with Invisalign Clear Aligners (CA). In total, 36 patients (16 males, 20 females, $9.9 \pm 1.9$ years) treated with CA are enrolled from the Department of Orthodontics. Inclusion criteria are the following: Caucasian ancestry, mixed dentition, molar Class II edge-to-edge, no tooth/craniofacial anomalies, no caries/periodontal diseases. Pre-treatment (T1) and post-treatment (T2) digital casts, and final ClinCheck representations (T2ClinCheck) are acquired. The Henry's angle (HA) is used to assess maxillary first molars rotation. The molars with an $\mathrm{HA}>11^{\circ}$ are taken (53 teeth). Five measurements are performed at T1, T2, and T2ClinCheck: Henry's angle (HA), mesiobuccal-expansion (MBE), distobuccal-expansion (DBE), mesiobuccal-sagittal (MBS), and distobuccal-sagittal (DBS). A paired $t$-test was used to compare $\mathrm{T} 2-\mathrm{T} 1$ and $\mathrm{T} 2 \mathrm{ClinCheck}-\mathrm{T} 2$. The T2-T1 shows a distal-rotation (difference $-6.3^{\circ}$ ) with an expansion of $2.2 \mathrm{~mm}$ for MBE and $1.5 \mathrm{~mm}$ for DBE. At T2, the mesiobuccal cusps show a distal movement of $1.0 \mathrm{~mm}$ and the distobuccal cusps of $0.9 \mathrm{~mm}$. The HA's T2ClinCheck-T2 difference is $-4.2^{\circ}$. In the sagittal plane, the difference is $0.9 \mathrm{~mm}$ for the MBS and $0.7 \mathrm{~mm}$ for the DBS. The expansion showed the highest predictability (60\% HA, 52.6\% MBS, and 56.25\% DBS). The CA effectively produces an arch expansion and upper molars' distal rotation. Upper molar derotation provides a $1 \mathrm{~mm}$ of gain in arch perimeter and occlusal improvement.
\end{abstract}

Keywords: class II malocclusion; clear aligner therapy; digital cast analysis

\section{Introduction}

The correct molar position and occlusal relationship have been described by Andrews as one of the six keys essential to achieving a successful orthodontic treatment [1,2]. In 1969, Ricketts described the following method to assess the proper distal rotations of the upper first molars in evaluating mesial drift in the malocclusion: in a parabolic dental arch form, the normal position of the upper first molar can be assessed when a line is drawn through the tips of the molar's disto-buccal and the mesio-lingual cusps cross the distal surface of the opposite canine [3]. Due to its rhomboidal shape with a wider buccolingual diameter than mesiodistal, a rotated upper first molar occupies more space, creating a situation unreceptive to obtain a normal occlusion with more crowding between adjacent teeth $[2,4]$. This rotation is caused by the mesiolingual cusp of the upper first molar, which firmly occludes in the central fossa of the lower first molar and acts as a center of rotation, causing a mesial movement of the mesiobuccal cusp [4]. The tooth occupies excessive space in the dental arch and its buccal cusps occlude with a tendency of Class II molar relationship [5]. 
In the literature, there are plenty of investigations focused on this topic [5-9]. The prevalence of the mesial rotation in subjects with the presence of Class II malocclusion is about $86 \%$. Lima et al. [6] found a correlation between the severity of Class II division 1 malocclusion and the amount of maxillary first molars' mesiopalatal rotation. Furthermore, Quinzi et al. reported that there is an association between upper first molar mesial rotation and anterior dental crowding in permanent dentition, while subjects in mixed dentition with mesial rotation should be considered at a higher risk of dental crowding [7]. In 2011, Giuntini et al. [8] investigated the upper first molar mesial rotation in Class II division 1 malocclusion subjects in mixed dentition. They performed dental cast measurements in a sample of 120 Class II division 1 subjects and in a sample of 58 Class I subjects, and they found that the amount of upper molar rotation was significantly greater in the Class II group than in the Class I group. In a recent case report, Giancotti et al. highlighted how maxillary molar derotation represented a strategic step to allow mesial mandibular shift and gain space in the arch, thus contributing to malocclusion correction [9].

However, in the literature there is a lack of information about the correction of upper first molar mesial rotation with clear aligners (CA).

The aim of the present study was to analyse the derotation movement of the maxillary mesio-rotated first permanent molars in subjects with Class II edge-to-edge dental malocclusion in mixed dentition treated with Invisalign Clear Aligners (CA).

\section{Materials and Methods}

This project was approved by the ethical committee of the University of Rome "Tor Vergata" (Protocol number: 163/20). All patients were aware of the study design and informed consent was obtained from patients' parents prior to participating.

In this prospective study, 36 patients (16 males, 20 females, mean age $9.9+/-1.9$ years) who received Invisalign system clear aligners treatment protocol (CA) were consecutively enrolled at the Department of Orthodontics of the University of Rome "Tor Vergata" from September 2019 to March 2021.

\subsection{Inclusion/Exclusion Criteria}

The patients were selected according to the following inclusion criteria: Caucasian ancestry, mixed dentition, Class II edge-to-edge first molar occlusal relationship assessed through clinical evaluation, and good compliance with aligners. The following exclusion criteria were applied: presence of second permanent molars, multiple and/or advanced caries, tooth agenesis, supernumerary teeth, cleft lip and/or palate, and other periodontal diseases.

\subsection{Therapeutic and Measurement Protocol}

For each patient, pre-treatment (T1) and post-treatment (T2) digital dental casts were taken using an intraoral scanner iTero ${ }^{\circledR}$ Orthodontic ver. 5.2.1.290 (Align Technology Inc., Santa Clara, CA, USA). Final position of the corresponding ClinCheck representation was also collected to establish the predictability of the final virtual model (T2ClinCheck) with respect to the movements observed in the post-treatment models.

On the initial digital casts, the upper permanent right and left first molars' rotations were assessed using the angular measurement of Henry [10] (Figure 1).

All the upper permanent first molars with a Henry's angle more than 11 degrees were analyzed in the study, making a sample of 53 teeth.

Patients received a non-extraction treatment protocol consisting of Invisalign System clear aligners.

The ClinCheck for each patient was planned with the same standardized disto-rotation protocol, according to Rickett's line [3]. Using ClinCheck software, a mesial-out buccal rotation of upper first molars was programmed with an amount of 2 degrees for each aligner. During the rotational correction, an arch expansion was always required. No distalization movements were included in the treatment protocol. 


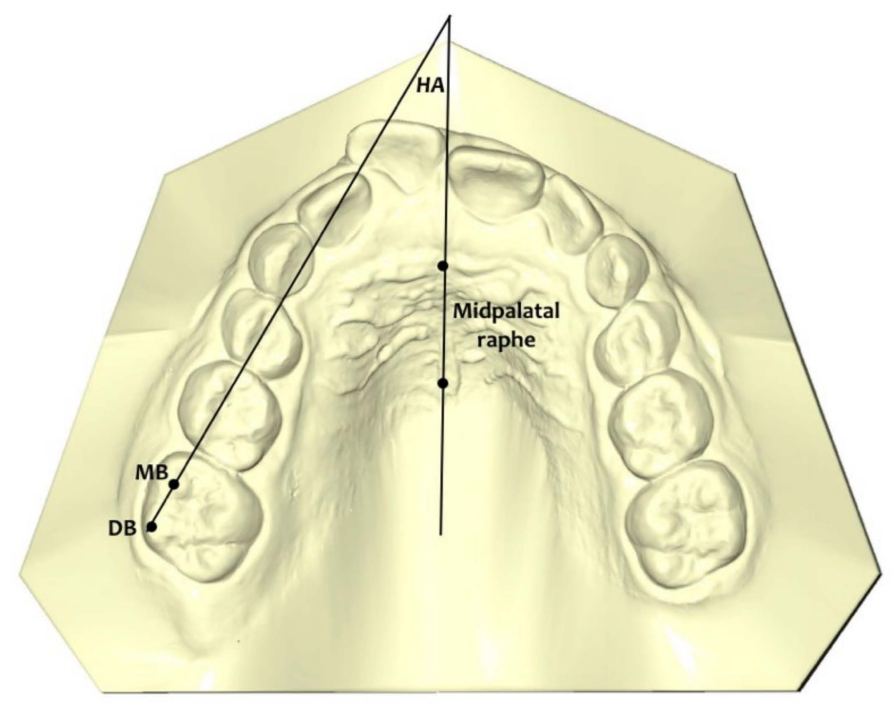

Figure 1. Henry's angle (HA) formed between the mid-palatal raphe and the line passing through the mesiobuccal (MB) and distobuccal (DB) cusps of the maxillary first molar.

All patients were instructed to wear their aligners full-time, excluding meals and tooth brushing. The patient changed the aligners every 7 days and every 4 stages the clinician checked for good aligner fitting and the position of the attachments. The mean number of aligners was 32 for the maxillary arch. If new scans were necessary to improve the fit of the aligners due to dental exfoliation and eruption, the prescription form was settled to continue the treatment until reaching the same final position as the first approved ClinCheck. Optimized expansion support attachments and optimized retention attachments were automatically placed by the software on the posterior teeth.

The average time between the initial and final scans was 8.6 months.

At the delivery appointment, the patients gained awareness that they were part of a research study, and honest compliance reporting was difficult. Patient's compliance was noted in the clinical diary. A single investigator conducted a face-to-face interview with each patient and their parents to assess his/her cooperation. Compliance was appraised on a 3-point Likert-type scale (poor, moderate, good) [11]. Poor compliance was reported when the patient wore the aligners less than $16 \mathrm{~h} /$ day; moderate from 16-20 h/day; good when the patient wore the aligner full time as suggested by the clinicians.

All the maxillary digital dental casts were exported in .stl format and for each of the 53 upper permanent first molars included in the study the following measurements were carried out at T1, T2, and T2ClinCheck using Viewbox 4 (dHAL software, Kifissia, Greece):

- Henry's angle (HA): the angle formed between the mid-palatal raphe (identified by an anterior and a posterior point along the mid-palatal raphe) and the line passing through the mesiobuccal (MB) and distobuccal (DB) cusps of the maxillary first molar [8] (Figure 1);

- Mesiobuccal Expansion (MBE): the perpendicular distance between the mesiobuccal cusp of the first right or/and left permanent molar and midpalatal raphe (Figure 2a);

- Distobuccal Expansion (DBE): the perpendicular distance between the distobuccal cusp of the first right or/and left permanent molar and midpalatal raphe (Figure 2a);

- Mesiobuccal Sagittal (MBS): the distance between the midpoint of the first palatal rugae and the projection of the mesiobuccal cusp of the first right or/and left permanent molar on the midpalatal raphe (Figure 2b);

- Distobuccal Sagittal (DBS): the distance between the midpoint of the first palatal rugae and the projection of the distobuccal cusp of the first right or/and left permanent molar on the midpalatal raphe (Figure $2 b$ ). 


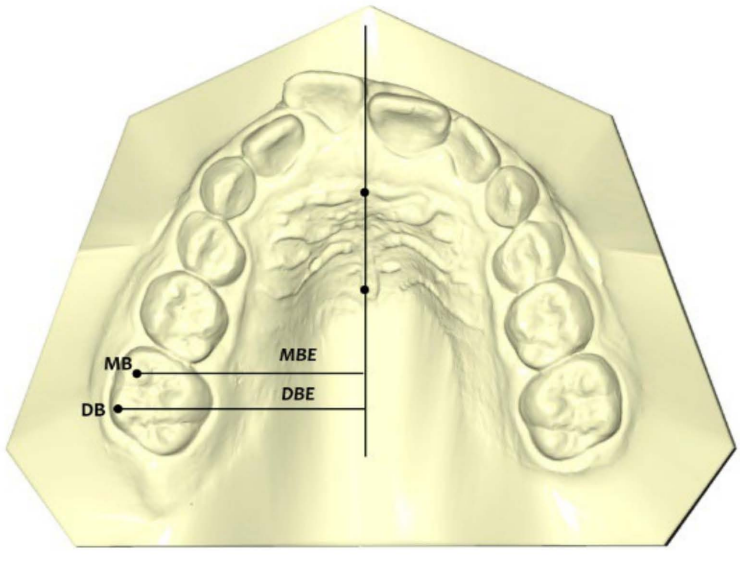

(a)

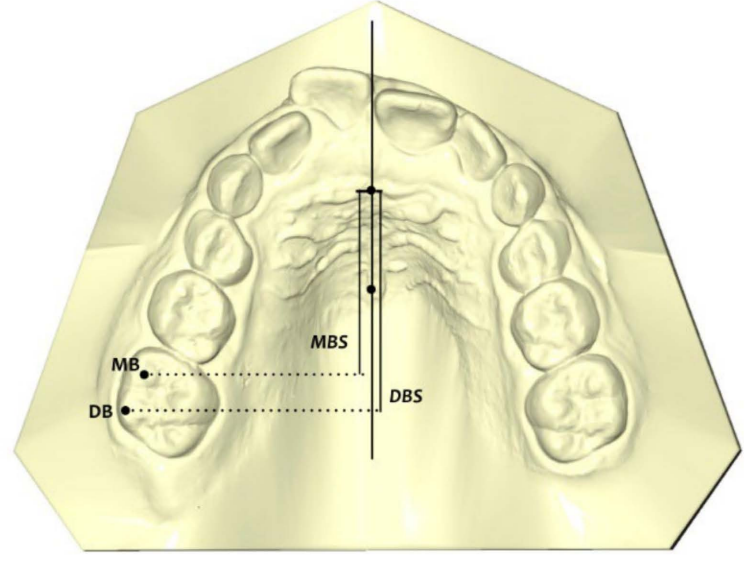

(b)

Figure 2. (a) Linear measurements of the expansion (MBE, DBE) movements of the upper first molar mesiobuccal (MB) and distobuccal (DB) right or/and left cusps. (b) Linear measurements of the distalization (MBS, DBS) movements of the upper first molar mesiobuccal (MB) or/and distobuccal (DB) right and left cusps.

\section{Statistical Analysis}

In a pilot study, 12 upper first permanent molars were used to calculate the reproducibility and the sample size, which indicated the need for approximately 45 teeth to estimate Henry's angle with a 95\% confidence interval (CI), a minimum difference of $2.3^{\circ}$ and a standard deviation (SD) of $3.5^{\circ}$, with a power of $80 \%$.

A unique operator (F.C.D.R.) performed all the measurements. In order to test the intra-examiner reliability, the same operator measured the entire sample again 2 weeks after the first assessment. The reliability of the measure was assessed by means of an interclass correlation coefficient (ICC).

Sample normality was tested by the Shapiro-Wilk test.

In the presence of normally distributed data, a paired $t$-test was selected to compare the T2-T1 changes and the T2ClinCheck-T2 differences. The level of significance was set at $5 \%$.

The Statistical Package for the Social Sciences (SPSS), version 18.0 (IBM Corp, Chicago, IL, USA) was the chosen software to analyze data.

\section{Results}

Of the 72 analyzed molars, 53 showed increased mesial rotation according to a Henry's angle of more than 11 degrees.

The analysis of compliance of the treated subjects (use of CA) showed that cooperation was good/moderate in all treated patients.

The ICC test showed almost perfect agreement with a score of 0.97 for the angular measurement and 0.96 for the linear measurements.

A new scan to make additional aligners was needed for eight patients to improve the fit of the aligners and better incorporate the erupting premolars into the appliance.

\subsection{Changes between Pre-Treatment (T1) and Post-Treatment (T2)}

Table 1 summarizes the differences between pre-treatment conditions and final outcomes, showing that there were statistically significant differences for all the performed measurements. In particular, the comparison between T2-T1 angular outcomes showed a distal rotation of the maxillary first permanent molars with a mean difference of $-6.3^{\circ}$ (mean HA T1: $17.5^{\circ} \pm 4.2^{\circ}$; mean HA T2: $11.2^{\circ} \pm 5.7^{\circ} ; p<0.001$ ). The mean transversal expansion was $2.2 \mathrm{~mm}$ for the mesiobuccal cusps (mean MBE T1: $24.3 \pm 1.0 \mathrm{~mm}$; mean 
MBE T2: $26.5 \pm 0.8 \mathrm{~mm} ; p<0.001$ ) and $1.5 \mathrm{~mm}$ for the distobuccal cusps (mean DBE T1: $26.1 \pm 0.9 \mathrm{~mm}$; mean DBE T2: $27.6 \pm 0.8 ; p<0.001)$. Regarding the sagittal plane, at T2, the mesiobuccal cusps showed an average distal movement of $1.0 \mathrm{~mm}$ (mean MBS T1: $29.3 \pm 2.2 \mathrm{~mm}$; mean MBS T2: $30.3 \pm 2.5 \mathrm{~mm} ; p<0.001)$, while the distobuccal cusps moved distally by a mean of $0.9 \mathrm{~mm}$ (mean DBS T1: $24.3 \pm 2.1 \mathrm{~mm}$; mean DBS T2: $25.2 \pm 2.5 \mathrm{~mm}$; $p<0.001)$.

Table 1. Descriptive statistics and statistical comparisons of T2-T1 changes (Paired $t$-test).

\begin{tabular}{|c|c|c|c|c|c|c|c|}
\hline \multirow[t]{2}{*}{ Measurements } & \multicolumn{2}{|c|}{ Pre-Treatment T1 $(n=53)$} & \multicolumn{2}{|c|}{ Post-Treatment T2 $(n=53)$} & \multirow[b]{2}{*}{ Diff } & \multirow[b]{2}{*}{$95 \% \mathrm{CI}$} & \multirow[b]{2}{*}{$p$ Value } \\
\hline & Mean & SD & Mean & SD & & & \\
\hline $\begin{array}{c}\text { Henry's } \\
\text { angle (HA) }\end{array}$ & 17.5 & 4.2 & 11.2 & 5.7 & -6.3 & $\begin{array}{c}-8.526 \text { to } \\
-4.165\end{array}$ & $* * *$ \\
\hline \multicolumn{8}{|l|}{ Mesiobuccal } \\
\hline $\begin{array}{l}\text { Expansion } \\
\text { (MBE) }\end{array}$ & 24.3 & 1.0 & 26.5 & 0.8 & 2.2 & 1.905 to 2.511 & $* * *$ \\
\hline \multicolumn{8}{|l|}{ Distobuccal } \\
\hline $\begin{array}{l}\text { Expansion } \\
\text { (DBE) }\end{array}$ & 26.1 & 0.9 & 27.6 & 0.8 & 1.5 & 1.255 to 1.779 & $* * *$ \\
\hline \multicolumn{8}{|l|}{ Mesiobuccal } \\
\hline $\begin{array}{c}\text { Sagittal } \\
\text { (MBS) }\end{array}$ & 29.3 & 2.2 & 30.3 & 2.5 & 1.0 & 1.431 to 0.636 & $* * *$ \\
\hline $\begin{array}{l}\text { Distobuccal } \\
\text { Sagittal } \\
\text { (DBS) }\end{array}$ & 24.3 & 2.1 & 25.2 & 2.5 & 0.9 & $\begin{array}{c}1.250 \text { to } \\
-0.475\end{array}$ & $* * *$ \\
\hline
\end{tabular}

SD: standard deviation; diff: mean difference; CI: confidence interval; ${ }^{* * *}=p<0.001$.

\subsection{Comparison between Post-Treatment (T2) and Final ClinCheck (T2ClinCheck) Results}

The predictability of ClinCheck of the Invisalign software was determined at the end of the treatment (Table 2) by establishing comparisons between the final molar rotations achieved in the digital models (T2) and the planned one (T2ClinCheck). A statistically significant difference was detected, meaning that the amount of change planned was not associated with the obtained outcomes. The mean difference between T2ClinCheck-T2 was $-4.2^{\circ}$ (mean HA T2: $11.2^{\circ} \pm 5.7^{\circ}$; mean HA T2ClinCheck: $7.0^{\circ} \pm 5.4^{\circ} ; p<0.001$ ). Comparable results were obtained for the measurements on the sagittal plane, showing a mean difference of $0.9 \mathrm{~mm}$ for the mesiobuccal cusps (mean MBS T2: $30.3 \mathrm{~mm} \pm 2.5 \mathrm{~mm}$; mean MBS T2ClinCheck: $31.2 \mathrm{~mm} \pm 2.3 \mathrm{~mm} ; p<0.001$ ) and $0.7 \mathrm{~mm}$ for the distobuccal cusps (mean DBS T2: $25.2 \pm 2.5 \mathrm{~mm}$; mean DBS T2ClinCheck: $25.9 \pm 2.2 \mathrm{~mm} ; p<0.001$ ). On the contrary, the expansion movements showed high predictability for both the mesiobuccal and distobuccal cusps. The mean difference between T2ClinCheck-T2 was $0.4 \mathrm{~mm}$ for $\mathrm{MBE}$ and $0.3 \mathrm{~mm}$ for DBE, meaning no statistically significant changes. As a percentage, predictability was $60 \%$ for Henry's angle, $52.6 \%$ for mesiobuccal sagittal movement, and $56.30 \%$ for distobuccal sagittal movement (Table 3 ). 
Table 2. Descriptive statistics and statistical comparisons of T2ClinCheck-T2 (Paired $t$-test).

\begin{tabular}{|c|c|c|c|c|c|c|c|}
\hline \multirow[t]{2}{*}{ Measurements } & \multicolumn{2}{|c|}{ Post-Treatment T2 $(n=53)$} & \multicolumn{2}{|c|}{ T2 ClinCheck $(n=53)$} & \multirow[b]{2}{*}{ Diff } & \multirow[b]{2}{*}{$95 \%$ CI } & \multirow[b]{2}{*}{$p$ Value } \\
\hline & Mean & SD & Mean & SD & & & \\
\hline $\begin{array}{l}\text { Henry's } \\
\text { angle (HA) }\end{array}$ & 11.2 & 5.7 & 7.0 & 5.4 & -4.2 & $\begin{array}{c}-5.289 \text { to } \\
-3.003\end{array}$ & $* * *$ \\
\hline Mesiobuccal & & & & & & & \\
\hline $\begin{array}{c}\text { Expansion } \\
\text { (MBE) }\end{array}$ & 26.6 & 0.8 & 27.0 & 0.7 & 0.4 & 0.154 to 0.780 & NS \\
\hline $\begin{array}{l}\text { Distobuccal } \\
\text { Expansion } \\
\text { (DBE) }\end{array}$ & 27.6 & 0.8 & 27.9 & 0.9 & 0.3 & $\begin{array}{c}-0.045 \text { to } \\
0.636\end{array}$ & NS \\
\hline $\begin{array}{l}\text { Mesiobuccal } \\
\text { Sagittal } \\
\text { (MBS) }\end{array}$ & 30.3 & 2.5 & 31.2 & 2.3 & 0.9 & 1.223 to 0.594 & $* * *$ \\
\hline $\begin{array}{l}\text { Distobuccal } \\
\text { Sagittal } \\
\text { (DBS) }\end{array}$ & 25.2 & 2.5 & 25.9 & 2.2 & 0.7 & $\begin{array}{c}1.032 \text { to } \\
-0.343\end{array}$ & $* * *$ \\
\hline
\end{tabular}

SD: standard deviation; diff: mean difference; CI: confidence interval; NS: Not Significant; ${ }^{* * *}=p<0.001$.

Table 3. Predictability of the changes: T2-T2ClinCheck.

\begin{tabular}{cc}
\hline Variable & Percentage $\%$ \\
\hline Henry's angle (HA) & $60 \%$ \\
Mesiobuccal Sagittal (MBS) & $52.60 \%$ \\
Distobuccal Sagittal (DBS) & $56.30 \%$ \\
\hline
\end{tabular}

\section{Discussion}

The purpose of the present study was to evaluate the changes in upper molar position and to assess the predictability of digital planning in a sample of growing patients with late mixed dentition or early permanent dentition with mesio-rotated upper molars treated with Invisalign CA.

In 1956, Henry stated, "The upper first permanent molars in the malocclusions are rotated in $83 \%$ of the cases, and the axis of rotation passes through the palatal root and the mesiolingual cusp" [10].

Several studies reported a high percentage of rotated maxillary first molars in Class II malocclusion. The upper first molar rotation existed in $85 \%$ of all orthodontic conditions, $90 \%$ of all Class II division 1 cases, and $100 \%$ of all maxillary constrictions [12]. It appeared that a transverse deficiency of the maxillary arch is a typical finding in growing subjects with upper molar mesial rotation [13]. Maxillary first molar rotates mesiolingually on its long axis, with the mesiolingual cusp and the lingual root acting as a center or axis rotation [13]. From the anatomical aspect, the upper first molar is prone to rotation due to its trapezoidal configuration and its triple roots, and thus the mesiopalatal cusp is a pivotal axis around which the upper first molar rotates [14]. For this reason, when digital planning the new molar position, it is important to apply the mesial-out buccal rotation, allowing for a distal rotation on the mesiopalatal cusp as a center of rotation. Moreover, derotation of the maxillary first molar while applying a simultaneous expansion force can provide centers of rotation at various points migrating from the molar center of resistance toward the distal margin ridge. The greater the expansion, the more the center of rotation will migrate distally. Therefore, the primary objective of accommodating a functional intercuspation is to expand and derotate at the same time [12].

Our results showed that there were statistically significant differences between T2-T1 maxillary first molar rotation (Figure 3). In the present study, the upper molars were mildly mesial rotated since the study group involved was in mixed dentition, before the exfoliation of second deciduous molars, and with a Class II edge-to-edge malocclusion. This meant that our treatment protocol required molar disto-rotation without distalization. The main 
derotation observed was of about six degrees. Giuntini et al. [8] reported that in the case of extreme mesial rotation of the upper permanent molar, i.e., when the molar is included in the dental arch perimeter with its oblique diameter instead of its mesiodistal diameter, maximal molar derotation leads to a corresponding maximal gain of space of about $2.5 \mathrm{~mm}$. According to our results, orthodontic molar derotation (about six degrees) led to a mean gain of arch space of about $1 \mathrm{~mm}$. Our findings agree with Braun et al. [12], who reported a value of gain in arch perimeter of $2.5 \mathrm{~mm}$ with an upper molar derotation of about 20 degrees. It is important to emphasize that the gain in arch perimeter following molar derotation occurred anteriorly to the derotated tooth, as the buccal cusps moved distally by about $1 \mathrm{~mm}$, with a simultaneous improvement in the molar occlusal relationship.

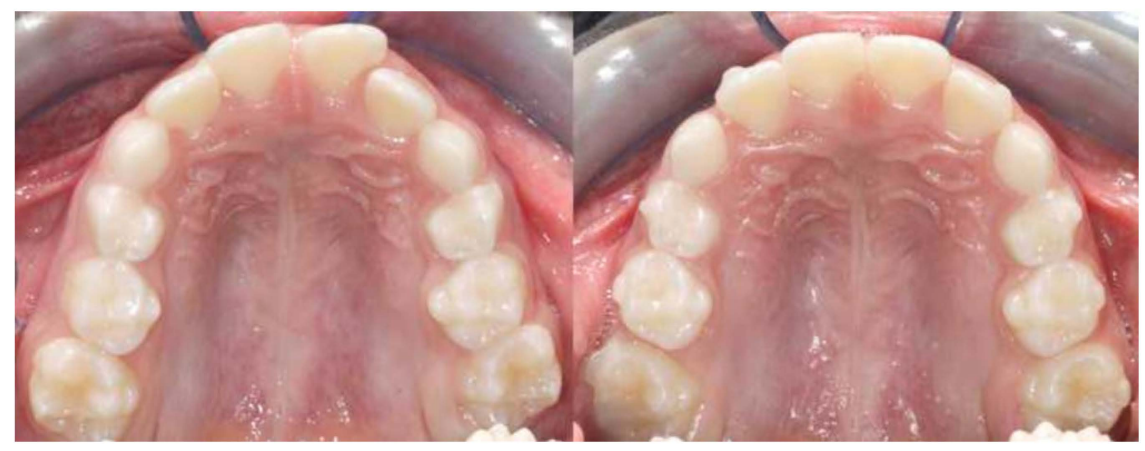

Figure 3. Intraoral occlusal evaluation pre-treatment (T1) and post-treatment (T2).

Our findings indicated that there were statistically significant differences between T2 effective rotation and T2ClinCheck programmed outcomes. ClinCheck software overestimated the value obtained in planning molar derotations. To test the predictability of the ClinCheck treatment planning software, measurements taken at the end of treatment (T2) were analyzed in comparison with the amount of movement predicted by the software at the end of the first phase of aligners (T2ClinCheck). The percentage of predictability of distal rotation was $60 \%$. These results are similar to those obtained by several studies [15-19], reporting that the ClinCheck generated a prognosis of greater distal rotation than actually realized. In a recent study, Haouili et al. [20] assessed that the mesial rotation of the upper first molars was around $42.9 \%$. The difference in percentage predictability could be associated with maxillary arch expansion, which is essential to gain further space and increase the predictability of disto-rotational movement. However, the rotation accuracy achieved in the present study was inferior when compared with the one reported by Morales-Burruezo et al. [15]. This difference is due to the fact that the predictability was tested at the end of the first set of aligners before any refinement.

Regarding the expansion movements, recently, Lione et al. [21] found a high predictability of the planned molar expansion with CA in permanent dentition. Our results showed that the same accuracy could be obtained in mixed dentition as well. Our data indicated that Invisalign CA are an effective tool for achieving transverse expansion as no statistical differences with the ClinCheck prediction were observed.

This is, in the literature, the first prospective investigation to compare the T2-T1 changes of the maxillary first permanent mesio-rotated molar position obtained by means of the Invisalign First System with the same disto-rotation protocol in all subjects with mixed dentition. Prior published data included case reports, material studies, technical articles, editorials, surveys, and studies assessing the predictability of multiple tooth movements. A limitation of the present investigation is its short-term nature and the small sample size of the treated groups. Therefore, further evaluations are necessary to increase the sample size and to analyze the stability of the results obtained in the long term. 


\section{Conclusions}

The outcomes of the present study revealed that subjects with Class II edge-to-edge mixed dentition often presented with mesial upper first molar rotation. In fact, of the 72 analyzed molars, 53 teeth had a moderate increase in mesial rotation. Molar distorotation is a complex movement involving expansion and distal drift of buccal cusps. Despite the complexity, CA is an effective tool for producing upper distal molar rotation with an observed $60 \%$ predictability. The orthodontic correction of mesial upper molar rotation provided $1 \mathrm{~mm}$ of gain in arch perimeter and improvement in molar relationships, with the movement of transversal expansion that showed the highest accuracy.

Author Contributions: Conceptualization, P.C. and R.L.; methodology, V.P. and R.L.; software, V.P.; validation, P.C., R.L. and C.P.; formal analysis, V.P.; investigation, F.C.D.R.; resources, C.P. and R.L.; data curation, F.C.D.R.; writing — original draft preparation, F.C.D.R.; writing—review and editing, R.L.; visualization, C.P.; supervision, C.P.; project administration, P.C. All authors have read and agreed to the published version of the manuscript.

Funding: No funding was received for conducting this study.

Institutional Review Board Statement: The study was conducted according to the guidelines of the Declaration of Helsinki, and approved by the Ethics Committee of the University of Rome "Tor Vergata" (protocol number 163/20, approved in December 2020).

Informed Consent Statement: Informed consent was obtained from all participants parents included in the study.

Data Availability Statement: The data presented in this study are available on request from the corresponding author.

Conflicts of Interest: The authors declare no conflict of interest.

\section{Abbreviations}

CA: Clear Aligners; HA: Henry's angle; MB: Mesiobuccal cusp of the upper first permanent molar; DB: Distobuccal cusp of the upper first permanent molar; MBE: Mesiobuccal-expansion; DBE: Distobuccalexpansion; MBS: Mesiobuccal-sagittal; DBS: Distobuccal-sagittal; T1: pre-treatment phase; T2: posttreatment phase; T2ClinCheck: final position of the ClinCheck representation; IC (confidence interval); SD (standard deviation); diff: mean difference; ICC (interclass correlation coefficient).

\section{References}

1. Angle, E.H. The classification of malocclusion. Dent. Cosm. 1899, 41, 248-264.

2. Andrews, L.F. The six keys to normal occlusion. Am. J. Orthod. 1972, 62, 296-309. [CrossRef]

3. Ricketts, R.M. Occlusion-The medium of dentistry. J. Prosthet. Dent. 1969, 21, 39-60. [CrossRef]

4. Dewel, B.F. Clinical observations on the axial inclination of teeth. Am. J. Orthod. 1949, 35, 98-115. [CrossRef]

5. Dahlquist, A.; Gebauer, U.; Ingervall, B. The effect of a transpalatal arch for the correction of first molar rotation. Eur. J. Orthod. 1996, 18, 257-267. [CrossRef]

6. Lima, B.P.; Pinzan-Vercelino, C.R.; Dias, L.S.; Bramante, F.S.; Tavarez, R.R. Correlation between the Rotation of the First Molars and the Severity of Class II Division 1 Malocclusion. Sci. World J. 2015, 2015, 261485. [CrossRef]

7. Quinzi, V.; Tecco, S.; Nota, A.; Caggiati, E.; Mummolo, S.; Marzo, G. Mesial Rotation of the Upper First Molar: Association with Anterior Dental Crowding in Mixed and Permanent. Appl. Sci. 2020, 10, 5301. [CrossRef]

8. Giuntini, V.; Baccetti, T.; Defraia, E.; Cozza, P.; Franchi, L. Mesial rotation of upper first molars in Class II division 1 malocclusion in the mixed dentition: A controlled blind study. Prog. Orthod. 2011, 12, 107-113. [CrossRef] [PubMed]

9. Giancotti, A.; Mampieri, M. Treatment staging in Class II, division 1 malocclusion correction: The importance of maxillary molar derotation. J. Aligner Ortho. 2020, 4, 237-246.

10. Henry, R.G. Relationship of the maxillary first permanent molar in normal occlusion and malocclusion: An intraoral study. Am. J. Orthod. 1956, 42, 288-306. [CrossRef]

11. Arnold, W.E.; McCroskey, J.C.; Prichard, S.V.O. The Likert-type scale. Today's Speech 1967, 15, 31-33. [CrossRef]

12. Braun, S.; Kusnoto, B.; Evans, C.A. The effect of maxillary first molar derotation on arch length. Am. J. Orthod. Dentofac. Orthop. 1997, 112, 538-544. [CrossRef]

13. Lamons, F.F.; Holmes, C.W. The problem of the rotated maxillary first permanent molar. Am. J. Orthod. 1961, 47, $246-272$. [CrossRef] 
14. Liu, D.; Melsen, B. Reappraisal of Class II molar relationships diagnosed from the lingual side. Clin. Orthod. Res. 2001, 4, 97-104.

15. Morales-Burruezo, I.; Gandía-Franco, J.L.; Cobo, J.; Vela-Hernández, A.; Bellot-Arcís, C. Arch expansion with the Invisalign system: Efficacy and predictability. PLoS ONE 2020, 15, e0242979. [CrossRef]

16. Boyd, R.L.; Waskalic, V. Three-dimensional Diagnosis and Orthodontic Treatment of Complex Malocclusions with the Invisalign Appliance. Semin. Orthod. 2001, 7, 274-293. [CrossRef]

17. Kassas, W.; Al-Jewair, T.; Preston, C.B.; Tabbaa, S. Assessment of Invisalign treatment outcomes using the ABO Model Grading System. J. World Fed. Orthod. 2013, 2, e61-e64. [CrossRef]

18. Ramis, M.R.; Gandia, V.; Bellot-Arcís, C.; Cibrian, R.; Peredes-Gallardo, V.; Gandia, J.L. Evaluation of Invisalign treatment using the Peer Assessment Rating (PAR) index. J. Aligner Ortho. 2018, 2, 13-19.

19. Iliadi, A.; Koletsi, D.; Eliades, T. Forces and moments generated by aligner-type appliances for orthodontic tooth movement: A systematic review and meta-analysis. Orthod. Craniofac. Res. 2019, 22, 248-258. [CrossRef]

20. Haouili, N.; Kravitz, N.D.; Vaid, N.R.; Ferguson, D.J.; Makki, L. Has Invisalign improved? A prospective follow-up study on the efficacy of tooth movement with Invisalign. Am. J. Orthod. Dentofacial Orthop. 2020, 158, 420-425. [CrossRef]

21. Lione, R.; Paoloni, V.; Bartolommei, L.; Gazzani, F.; Meuli, S.; Pavoni, C.; Cozza, P. Maxillary arch development with Invisalign system. Angle Orthod. 2021, 91, 433-440. [CrossRef] [PubMed] 